\title{
7. THE WHITE DWARFS IN THE CATALOGUE OF NEARBY STARS OF 1969*
}

\author{
W. GLIESE \\ Astronomisches Rechen-Institut, Heidelberg, Germany
}

The Catalogue of Nearby Stars (Gliese, 1969) contains 1529 single stars and systems with altogether 1890 components. It includes 54 white dwarfs with apparent magnitudes from 8.5 to 17 ; namely 38 single stars and 16 degenerate stars which are components of binaries or triples. Only 48 of these objects have parallaxes larger than 0.050 . But our knowledge is very incomplete.

The distances of 33 of these white dwarfs have been determined trigonometrically with probable errors (p.e.) between $2 \%$ and $30 \%, 4$ distances are from the photometric parallaxes of the companions, one star is a Hyades member, another belongs to the Wolf 219 group (group parallax), and for 15 objects the absolute magnitudes have been taken from the $\left(M_{V}, \mathrm{U}-\mathrm{V}\right)$ relations by Eggen and Greenstein (1965).

The absolute magnitudes of 18 degenerate stars are known with probable errors smaller than $\pm 0 \mathrm{~m} \cdot 21$, but only for 8 of these objects have $\mathrm{U}, \mathrm{B}, \mathrm{V}$ photometry and space velocities also been determined. This demonstrates the incompleteness of our knowledge.

Nevertheless I shall try to point to some questions which may be indicated by this material.

In the considerations concerning velocities we exclude the DAss star LP 9-231 whose space velocity is nearly $350 \mathrm{~km} / \mathrm{sec}$.

(a) Radial Velocities. The radial velocities of 18 of these degenerate stars have been measured. After elimination of the standard solar motion the mean RV is +45 $\pm 4.5 \mathrm{~km} / \mathrm{sec}$ (p.e.), in good agreement with the mean Einstein effect of $51 \mathrm{~km} / \mathrm{sec}$ derived by Greenstein and Trimble (1967). The space velocities in the Nearby Star Catalogue have been computed after correcting the measured RV by $-51 \mathrm{~km} / \mathrm{sec}$.

The comparison of the measured RV's of $o^{2}$ Eri B and of CoD $-37^{\circ} 10500 \mathrm{~B}$ with that of their companions gives differences of $+22 \mathrm{resp} .+56 \mathrm{~km} / \mathrm{sec}$. The mass of $o^{2}$ Eri B is $0.43 M_{\odot}$ - probably somewhat smaller than the mean mass of a white dwarf.

(b) Proper Motions and Tangential Velocities. Normally parallax programs of faint stars prefer objects with large proper motions. Search for degenerate stars independent of their proper motions has so far been very limited.

The mean annual proper motion of the 33 white dwarfs with trigonometric parallaxes is 1."33 whereas the mean for the 15 stars with luminosities estimated by Eggen and Greenstein is only 0."39. Even if we should not jump to conclusions from this difference the enlistment of some of these objects with very small proper motions

* Mitteilungen Serie A, No. 43.

Luyten (ed.), White Dwarfs, 35-40. All Rights Reserved.

Copyright C 1971 by the IAU. 
in the Nearby Star Catalogue seems to be dubious: Feige 34, Feige 110, CPD $-69^{\circ} 177$, Feige 22, Case 1. The tangential velocities of these 5 objects relative to the sun are below $10 \mathrm{~km} / \mathrm{sec}$. Except Case 1, their luminosities derived from the $\left(M_{V}, \mathrm{U}-\mathrm{V}\right)$ relation of white dwarfs are fairly bright. In the group of white dwarfs with photometric distances they are responsible for an increasing mean tangential velocity (TV) with decreasing luminosity whereas the mean TV among the 32 objects with trigonometric parallaxes does not change remarkably with the absolute magnitude (Table I).

\section{TABLE I}

Luminosities and tangential velocities of white dwarfs

\begin{tabular}{|c|c|c|c|c|}
\hline \multirow[b]{2}{*}{$M_{V}$} & \multicolumn{2}{|c|}{ photom. parallax } & \multicolumn{2}{|c|}{ trigon. parallax } \\
\hline & $n$ & $\begin{array}{l}\overline{\mathrm{TV}} \\
\mathrm{km} / \mathrm{sec}\end{array}$ & $n$ & $\begin{array}{l}\overline{\mathrm{TV}} \\
\mathrm{km} / \mathrm{sec}\end{array}$ \\
\hline $10-11$ & 5 & 7 & 1 & 44 \\
\hline $11-12$ & & & 5 & 65 \\
\hline $12-13$ & 5 & 26 & 6 & 38 \\
\hline $13-14$ & 4 & 39 & 7 & 43 \\
\hline $14-15$ & 1 & 103 & 7 & 70 \\
\hline $15-16$ & & & 6 & 73 \\
\hline
\end{tabular}

(c) Solar Motion. The space velocities of 17 white dwarfs - single stars or primaries in a system - give the solar motion and velocity dispersion shown in Table II. The measured RV's have been corrected by $-51 \mathrm{~km} / \mathrm{sec}$. The U-, V-, W-axes are in a

TABLE II

Solar motion and velocity dispersion $(\mathrm{km} / \mathrm{sec})$

\begin{tabular}{lcccrrrrrrr} 
Class & $n$ & $\mathrm{~V}_{\odot}$ & $(\mathrm{km} / \mathrm{sec})$ & $L_{a}$ & p.e. & $B_{a}$ & p.e. & $\sigma_{\mathrm{U}}$ & $\sigma_{\mathrm{V}}$ & \multicolumn{1}{c}{$\sigma_{\mathrm{W}}$} \\
$\mathrm{D}$ & 17 & 20 & \pm 4 & $69^{\circ}$ & \pm 20 & $+21^{\circ}$ & \pm 10 & \pm 42 & \pm 20 & 21 \\
$\mathrm{dM}$ & 202 & 23 & 1.3 & 71 & 5 & +16 & 3 & 38 & 25 & 21 \\
$\mathrm{dMe}$ & 106 & 13 & 1.3 & 66 & 10 & +31 & 6 & 31 & 19 & 13 \\
\hline
\end{tabular}

righthand galactic system, $\mathrm{U}$ directed to the galactic centre. The data are compared with the results of an investigation of the early $M$ dwarfs in the McCormick program (Gliese, 1958) which obviously represents the red dwarfs free from selection effects.

The velocity distribution of the few white dwarfs agrees fairly well with that of the $\mathrm{dM}$ stars. The deviations from the data of the emission-line red dwarfs seem to be larger. But in view of the large errors this result should not be over-valued.

If we do not correct the measured RV's of white dwarfs by $-51 \mathrm{~km} / \mathrm{sec}$ (mean Einstein effect) the dispersion among the space velocities of 16 stars is distinctly larger than the data in Table II: $\pm 53, \pm 26, \pm 30 \mathrm{~km} / \mathrm{sec}$. 
(d) Colour-Luminosity Relations. In Figures 1, 2, and 3 the distribution of the stars in the $\left(M_{V}, \mathrm{~B}-\mathrm{V}\right),\left(M_{V}, \mathrm{U}-\mathrm{B}\right)$, and the $\left(M_{V}, \mathrm{U}-\mathrm{V}\right)$ plane is shown. They include only the 15 objects whose trigonometrically determined absolute magnitudes are known with probable errors not exceeding \pm 0 m 20 . The size of the rectangles is defined by $M_{V}-5 \times$ p.e., $M_{V}+5 \times$ p.e. (maximal error of $M_{V}$ which results from the errors in the trigonometric parallax and in the apparent magnitude); the errors in the colour data are estimated values. So we may assume that the true position of each star will be within the limits of its rectangle.

The $\left(M_{V}, \mathrm{~B}-\mathrm{V}\right)$ diagram does not show much more than a real cosmic dispersion. In the $\left(M_{V}, \mathrm{U}-\mathrm{B}\right)$ plane the two groups pointed out by Eggen and Greenstein (1965) are indicated - the brighter group represented by only 4 stars. The same is true of the $\left(M_{V}, \mathrm{U}-\mathrm{V}\right)$ plane.

Obviously a star like $o^{2}$ Eri B belongs to another group than the stars of nearly the same colour but about two classes fainter. A real gap seems to be possible between both samples.

The addition of the stars with more inaccurate data does not give much more information but it obscures the answer to the question of the existence of a gap.

In the $(\mathrm{U}-\mathrm{B}, \mathrm{B}-\mathrm{V})$ diagram of these 15 objects (Figure 4) the 4 bright degenerate stars of the upper relation (relation I of Eggen and Greenstein, 1965) are farther below

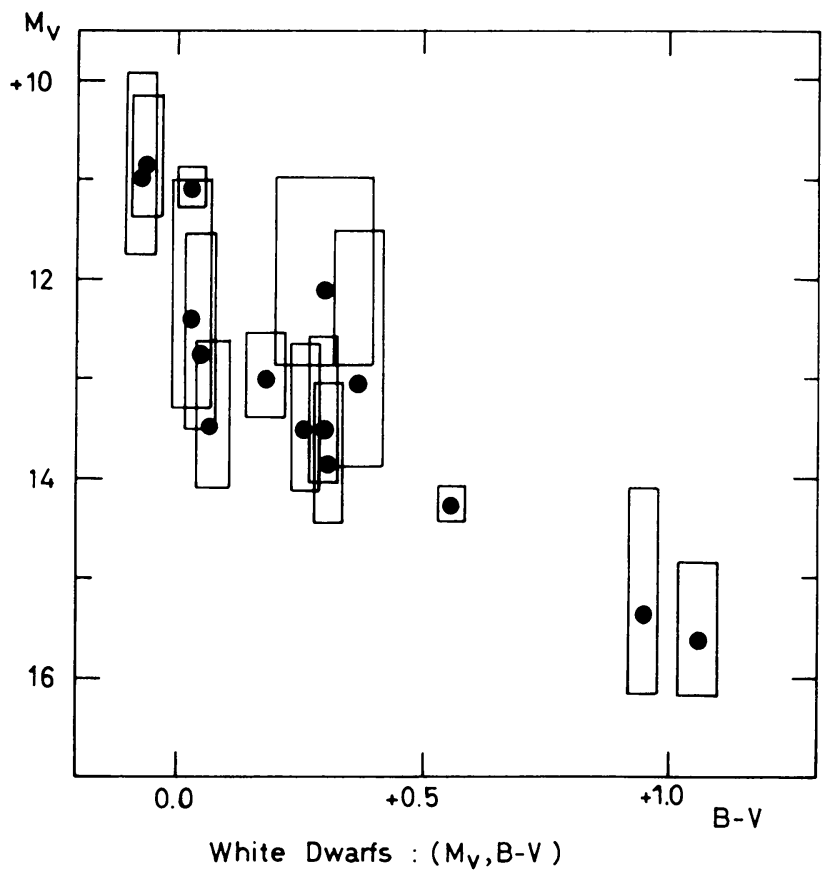

Fig. 1. The $\left(M_{v}, \mathrm{~B}-\mathrm{V}\right)$ diagram for white dwarfs with trigonometrically determined absolute magnitudes, p.e. not exceeding $\pm 0^{\mathrm{m}} .20$. The rectangles represent the areas whose limits indicate maximal errors (maximal error $=5 \times$ prob. error). 


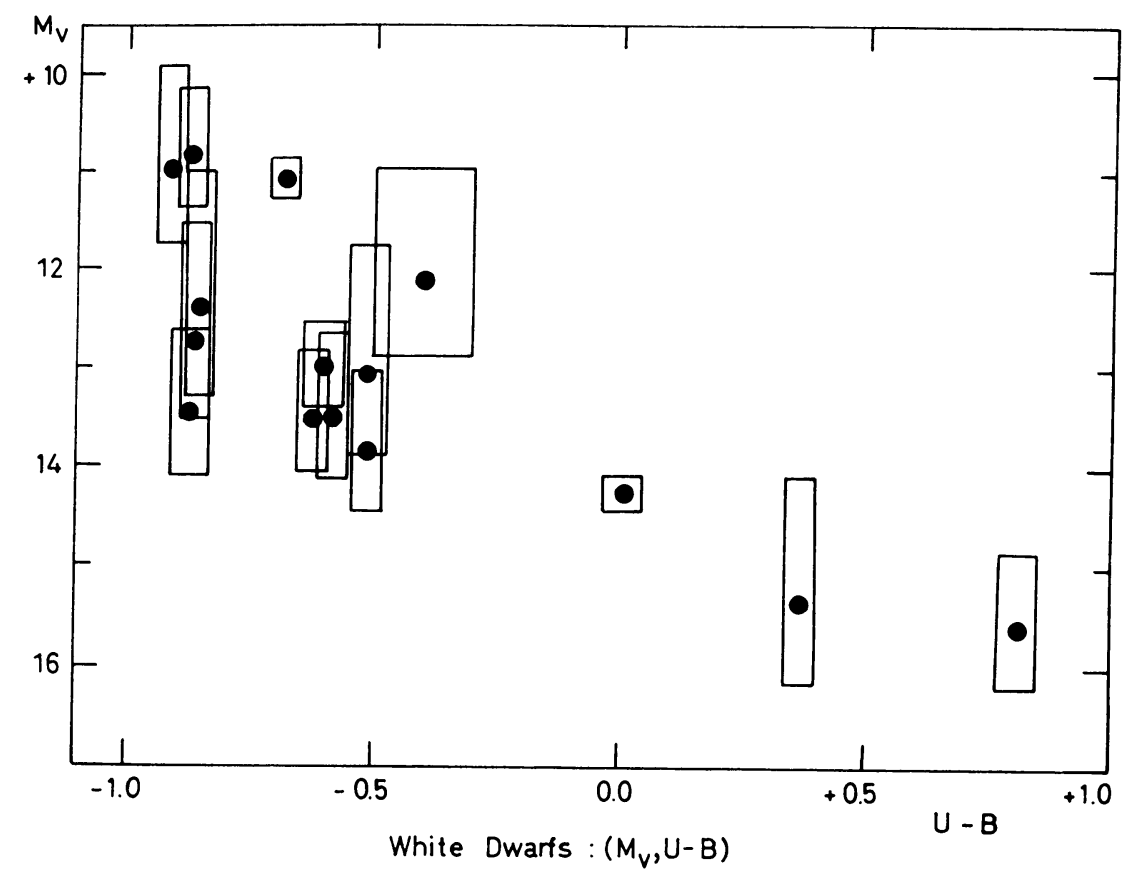

Fig. 2. The $\left(M_{V}, \mathrm{U}-\mathrm{B}\right)$ diagram for white dwarfs with trigonometrically determined absolute magnitudes, p.e. not exceeding $\pm 0^{\mathrm{m}} .20$. The rectangles represent the areas whose limits indicate maximal errors (maximal error $=5 \times$ prob. error).

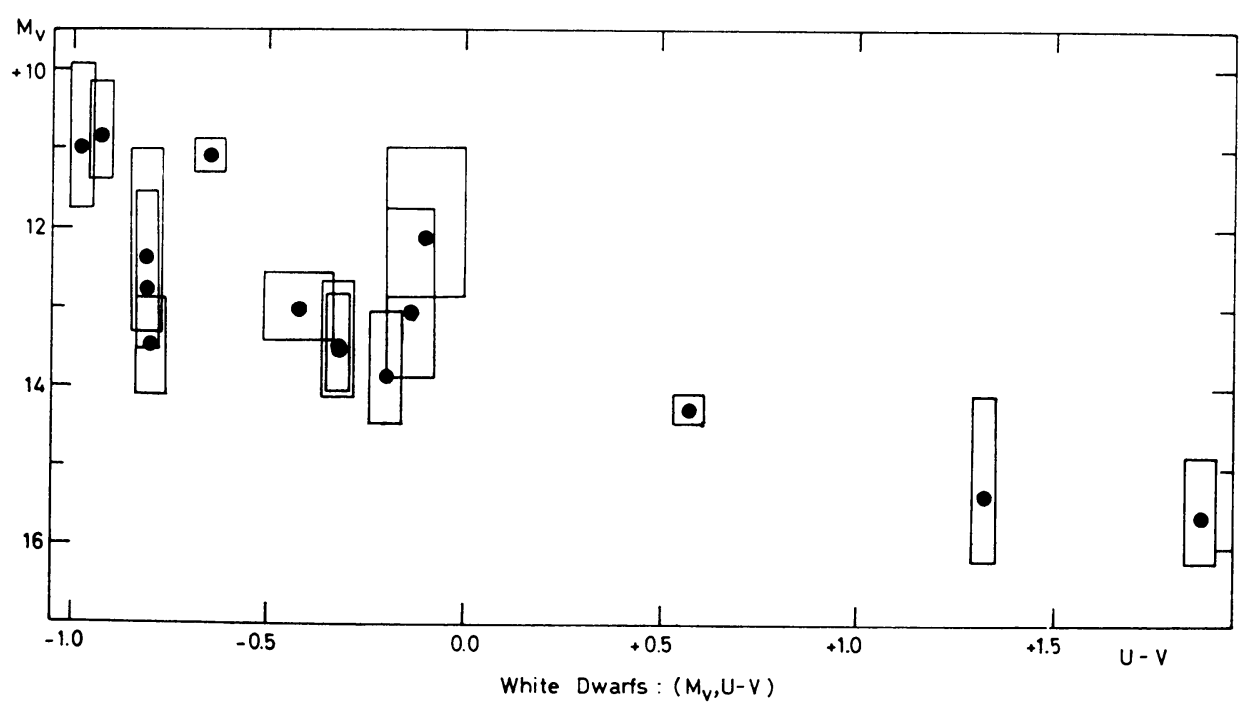

Fig. 3. The $\left(M_{V}, \mathrm{U}-\mathrm{V}\right)$ diagram for white dwarfs with trigonometrically determined absolute magnitudes, p.e. not exceeding $\pm 0^{\mathrm{m}} .20$. The rectangles represent the areas whose limits indicate maximal errors (maximal error $=5 \times$ prob. error). 




Fig. 4. Positions of the white dwarfs with well-known luminosities in the $(\mathrm{U}-\mathrm{B}, \mathrm{B}-\mathrm{V})$-plane. Open circles indicate the stars of bright luminosities (relation I, Eggen and Greenstein, 1965); the lowest of these 4 stars has only preliminary colour data. The continuous curve represents the black-body line.

the black-body line than the stars of the second relation. Probably there really is a slight difference between both groups of white dwarfs at least in the region of the A type stars.

(e) Binaries and Triples. Fourteen of the 54 nearby objects are components of double stars; two further white dwarfs belong to triples. In four systems the degenerate star is the primary. For Sirius, Procyon, and $o^{2}$ Eri the orbits and masses are known. If we estimate the masses of the other companions we may assume that in 8 or even 9 of the systems the white dwarf component will be the primary in mass.

Figure 5 shows the Hertzsprung-Russell diagram of the companions; nearly all of them are main-sequence stars, mostly red dwarfs. The 5 emission-line stars (open circles) are $o^{2}$ Eri A (K1 Ve) and C (dM5e), BD+26 730 (dK5ep) - which probably is a member of the Hyades group -, the eclipsing binary LP 101-15 (dM3e-dM4e), 


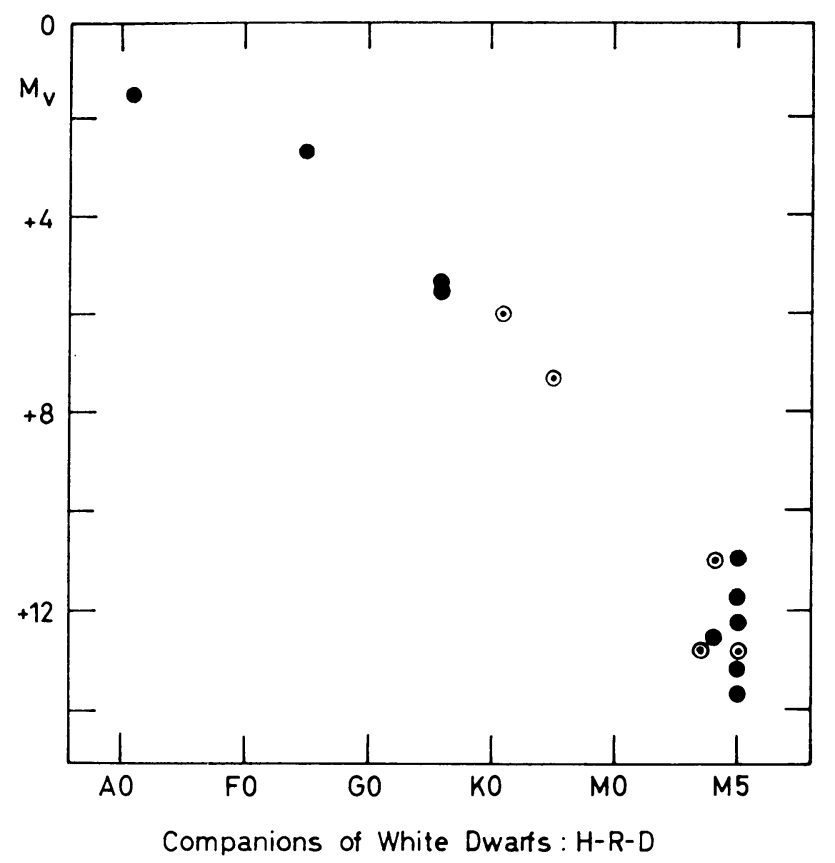

Fig. 5. H-R-D of the companions of white dwarfs. Emission-line stars are shown as open circles.

and Ross 193 (dM4e). The systems $+26^{\circ} 730 / \mathrm{G} 39-27$ and Ross 193/VB 11, together with the problem of a combination of a degenerate star with an emission-line red dwarf, have been discussed by Eggen and Greenstein (1967). $o^{2}$ Eri and LP 101-15/16 are high velocity systems.

This small sample of binaries does not show any striking correlations. The components are from A1 V (Sirius) resp. F5 IV-V (Procyon) down to dM5 or even later (L 745-46 B); the degenerate components are of type A, As, Awk, F, or C. The magnitude differences "white dwarf minus companion" are from 10.5 to -3.0 . The tangential distances are from $16 \mathrm{AU}$ (Procyon) to $9000 \mathrm{AU}$. But there is no correlation between distance and type or magnitude difference.

This short report on the degenerate stars with the best known distances, luminosities, and tangential velocities must close with the disappointing remark that these data are far from being sufficient for detailed discussions of their problems.

\section{References}

Eggen, O. J. and Greenstein, J. L.: 1965, Astrophys. J. 141, 83.

Eggen, O. J. and Greenstein, J. L.: 1967, Astrophys. J. 150, 927.

Gliese, W.: 1958, Z. Astrophys. 45, 293.

Gliese, W.: 1969, Veröff. Astron. Rechen Inst. Heidelberg 22.

Greenstein, J. L. and Trimble, V. L.: 1967, Astrophys. J. 149283. 